\title{
Joint Dimming Control and Transceiver Design for MIMO-Aided Visible Light Communication
}

\author{
Author 1, Author 2, Author 3, Author 4, Author 5
}

\begin{abstract}
The multiple-input multiple-output (MIMO) concept has been readily invoked in visible light communication (VLC) for increasing data rate. In this paper, we conceive a general solution of dimming control and MIMO transceiver design for VLC, which is capable of minimizing the mean-squared error between the transmitted and received signals, while at the same time, maintaining a specific indoor illumination level. We take into consideration practical optical constraints in the design, including the LED non-linearity and the specific dimming requirements. An efficient solution of our design problem is derived by conceiving a projected gradient algorithm. Our numerical results show that the proposed scheme achieves better bit error rate (BER) performance as well as significantly higher convergence speed than its benchmarker conceived in 2015.
\end{abstract}

Index Terms-Multiple-input multiple-output, visible light communication, transceiver, mean-squared error.

\section{INTRODUCTION}

Given the unsatiable customer demand for high-speed data transmission and the saturation of the radio frequency (RF) spectrum, visible light communication (VLC) is deemed to be an important supplement of $5 \mathrm{G}$ indoor wireless communications [1]. In VLC, the classic multiple-input multiple-output (MIMO) concept can be readily invoked for boosting the data rate. In order to improve the attainable link performance of MIMO systems, transmit precoding (TPC) techniques have been extensively studied in RF communications. However, VLC has different features from RF communications, such as the non-negative nature of signals and the all important illumination requirements [2]. Therefore, these algorithms cannot be directly applied to VLC. The distinctive features of VLC have inspired us to develop specific signal processing algorithms for MIMO-aided VLC.

Recently, there have been several important contributions on MIMO-aided VLC. Zeng et. al [3] investigated multiplexingoriented MIMO VLC systems relying both on imaging and non-imaging receivers. An experimental demonstration of MIMO VLC using a non-imaging receiver was presented by Burton et. al [4]. In [5], novel angle diversity receivers were proposed for increasing the throughput of MIMO VLC. However, all the above exciting contributions were focused on the device-design and on the associated experimental aspects. By contrast, Park et. al [6] proposed an adaptive MIMO transceiver for maximizing the data rate, but no dimming control was considered. In [7], a MIMO transceiver using zero-mean modulation was developed for VLC under the nonnegative constraint of the optical signals. Since zero-mean modulation schemes do not affect the average optical power, dimming control relies on adjusting the direct current (DC) offset to the target illumination level. But again, this is not applicable to the modulation associated with a non-zero mean.
Without restricting our attention to the special case of zeromean modulation, we propose a general solution of dimming control and MIMO transceiver design in VLC, for minimizing the mean-squared error (MSE) between the transmitted and received signals, while maintaining the specific indoor illumination level. The main contributions of the paper are:

- Our scheme is applicable to a wide range of modulation schemes, such as pulse amplitude modulation (PAM) and pulse position modulation (PPM).

- More explicitly, we take into account several practical optical constraints in our design, including the LED non-linearity and the dimming requirement, for avoiding the non-linear clipping of signals as well as to provide the target illumination level. - An efficient solution is derived by invoking the projected gradient (PG) algorithm, which yields a simple solution without requiring any special optimization software package [8]. Our numerical results demonstrate that the proposed scheme achieves better BER performance as well as higher convergence speed compared to [7].

\section{System Model and Optical Power Constraints}

We consider a MIMO VLC system composed of $N_{t}$ LED transmitters on the ceiling of the room and a receiver equipped with $N_{r}$ photodetectors (PDs). Let $\mathbf{s}=\left[s_{1}, \cdots, s_{K}\right]^{T}$ denote the real source data vector, where $s_{j}$ is a finite-magnitude signal bounded by $\Delta_{L, j} \leq s_{j} \leq \Delta_{H, j}$, and $K$ is the number of data streams. Given $\mathbf{s}$, the transmitted signal is expressed as $\mathbf{x}=\mathbf{W} \mathbf{s}+\mathbf{p}$, where $\mathbf{W} \in \mathbb{R}^{N_{t} \times K}$ is the TPC matrix, and $\mathbf{p}=\left[p_{1}, \cdots, p_{N_{t}}\right]^{\mathrm{T}}$ is the DC offset vector. Furthermore, the signal $x_{i}$ transmitted by the $i$ th LED is given by $x_{i}=\mathbf{w}_{i} \mathbf{s}=$ $\sum_{j=1}^{K} w_{i j} s_{j}+p_{i}$, where $\mathbf{w}_{i}$ is the $i$ th row of $\mathbf{W}$ and $w_{i j}$ is the element in the $i$ th column and $j$ th row of $\mathbf{W}$.

Due to the nonlinear LED transfer characteristic, the transmitted signal is constrained to a limited linear dynamic range, i.e., we have $p_{L, i} \leq x_{i} \leq p_{H, i}$, where $p_{L, i}$ and $p_{H, i}$ denote the minimum and maximum drive current permitted by the $i$ th LED, respectively [9]. By defining $\Delta_{j}=\left(\Delta_{H, j}-\Delta_{L, j}\right) / 2$ and $c_{j}=\left(\Delta_{H, j}+\Delta_{L, j}\right) / 2$, we have $-\Delta_{j} \leq s_{j}-c_{j} \leq \Delta_{j}$. Furthermore, we have $-\sum_{j=1}^{K}\left|w_{i j}\right| \Delta_{j} \leq \sum_{j=1}^{K} w_{i j}\left(s_{j}-c_{j}\right) \leq$ $\sum_{j=1}^{K}\left|w_{i j}\right| \Delta_{j}$ [6]. Thus, the dynamic range of $x_{i}$ is given by

$-\operatorname{abs}\left\{\mathbf{w}_{i}\right\} \boldsymbol{\Delta}+\mathbf{w}_{i} \mathbf{c}+p_{i} \leq x_{i} \leq \operatorname{abs}\left\{\mathbf{w}_{i}\right\} \boldsymbol{\Delta}+\mathbf{w}_{i} \mathbf{c}+p_{i}$,

where $\operatorname{abs}\{\cdot\}$ is the element-wise absolute value operator, $\boldsymbol{\Delta}=$ $\left[\Delta_{1}, \cdots, \Delta_{K}\right]^{T}$ and $\mathbf{c}=\left[c_{1}, \cdots, c_{K}\right]^{T}$. To ensure that $p_{L, i} \leq$ $x_{i} \leq p_{H, i}, \mathbf{w}_{i}$ should satisfy $-\operatorname{abs}\left\{\mathbf{w}_{i}\right\} \boldsymbol{\Delta}+\mathbf{w}_{i} \mathbf{c}+p_{i} \geq p_{L, i}$, 
and abs $\left\{\mathbf{w}_{i}\right\} \boldsymbol{\Delta}+\mathbf{w}_{i} \mathbf{c}+p_{i} \leq p_{H, i}$. Consequently, to guarantee that all LEDs operate within their limited linear dynamic range, the TPC matrix should satisfy

$$
\left\{\begin{array}{l}
\operatorname{abs}\{\mathbf{W}\} \boldsymbol{\Delta}-\mathbf{W} \mathbf{c} \leq \mathbf{p}-\mathbf{p}_{L} \\
\operatorname{abs}\{\mathbf{W}\} \boldsymbol{\Delta}+\mathbf{W} \mathbf{c} \leq \mathbf{p}_{H}-\mathbf{p}
\end{array},\right.
$$

where we have $\mathbf{p}_{L}=\left[p_{L, 1}, \cdots, p_{L, N_{t}}\right]^{T}$ and $\mathbf{p}_{H}=$ $\left[p_{H, 1}, \cdots, p_{H, N_{t}}\right]^{T}$. Furthermore, since most people cannot perceive the fluctuations of light signals as long as the frequency of modulation is above $100 \mathrm{~Hz}$, the average amplitude of the signals can be readily adjusted to provide the target brightness level. Mathematically, the dimming control of VLC requires [9]

$$
\mathrm{E}\{\mathbf{x}\}=\mathbf{W E}\{\mathbf{s}\}+\mathbf{p}=\mathbf{W b}+\mathbf{p}=\mathbf{p}_{T},
$$

where $\mathrm{E}\{\cdot\}$ denotes the statistical expectation, $\mathbf{b} \in \mathbb{R}^{K}$ is the mean of $\mathbf{s}$, and $\mathbf{p}_{T}=\left[p_{T, 1}, \cdots, p_{T, N_{t}}\right]^{T}$ is the average drive current of the LEDs required for the target dimming level. The dimming level of the $i$ th LED is defined as $\rho=$ $\left(p_{T, i}-p_{L, i}\right) /\left(p_{H, i}-p_{L, i}\right)$. From (3), we can observe that both $\mathbf{W}$ and $\mathbf{p}$ have to be adjusted simultaneously to achieve the target dimming level.

At the receiver, the light rays received by the PD are converted into electrical signals. After removing the DC offset, the received signal is processed by the linear equalizer represented by $\mathbf{G} \in \mathbb{R}^{K \times N_{r}}$ for recovering the original transmitted data, i.e., $\widehat{\mathbf{s}}=\mathbf{G}(\mathbf{H W} \mathbf{s}+\mathbf{n})$, where $\widehat{\mathbf{s}}$ is the detected data vector, $\mathbf{H} \in \mathbb{R}^{N_{r} \times N_{t}}$ is the channel matrix spanning from the LED array to the receiver PDs, and $\mathbf{n} \in \mathbb{R}^{N_{r}}$ is the additive white Gaussian noise (AWGN) having a covariance matrix of $\mathbf{R}_{n}$. The MSE between the detected symbols and the original transmitted symbols is expressed as

$$
\begin{aligned}
\mathrm{MSE} & =\operatorname{tr}\left\{\mathrm{E}\left[(\widehat{\mathbf{s}}-\mathbf{s})(\widehat{\mathbf{s}}-\mathbf{s})^{T}\right]\right\}=\operatorname{tr}\left\{\mathbf{R}_{s}+\mathbf{G R}_{n} \mathbf{G}^{T}\right. \\
& \left.-2 \mathbf{G H W} \mathbf{R}_{s}+\mathbf{G H W R} \mathbf{W}_{s} \mathbf{W}^{T} \mathbf{H}^{T} \mathbf{G}^{T}\right\},
\end{aligned}
$$

where $\operatorname{tr}\{\cdot\}$ denotes the trace of the matrix, while $\mathbf{R}_{s}$ is the covariance matrix of $\mathbf{s}$.

\section{Joint Dimming Control And Transceiver DESIGN}

In this section, we propose a joint dimming control and transceiver design method for our MIMO VLC system. The objective of our design is to minimize the MSE, as well as to maintain the target illumination level. Accordingly, the design problem can be formulated as

$$
\min _{\mathbf{G}, \mathbf{W}, \mathbf{p}} \text { MSE, s.t. (2), (3). }
$$

By reformulating the illumination constraint in (3), we have $\mathbf{p}=\mathbf{p}_{T}-\mathbf{W b}$. Thus, the optimization problem in (5) can be equivalently simplified to

$$
\min _{\mathbf{G}, \mathbf{W}} \text { MSE, }
$$

$$
\text { s.t. } \operatorname{abs}\{\mathbf{W}\} \boldsymbol{\Delta}+\mathbf{W} \widehat{\mathbf{b}} \leq \widehat{\mathbf{p}}_{L}, \operatorname{abs}\{\mathbf{W}\} \boldsymbol{\Delta}-\mathbf{W} \widehat{\mathbf{b}} \leq \widehat{\mathbf{p}}_{H},
$$

where we have $\widehat{\mathbf{b}}=\mathbf{b}-\mathbf{c}, \widehat{\mathbf{p}}_{L}=\mathbf{p}_{T}-\mathbf{p}_{L}$ and $\widehat{\mathbf{p}}_{H}=\mathbf{p}_{H}-\mathbf{p}_{T}$. We first optimize $\mathbf{G}$ by setting the derivative of the MSE in (4) with respect to (w.r.t.) $\mathbf{G}$ equal to zero, yielding:

$$
2 \mathbf{G}\left(\mathbf{H W} \mathbf{R}_{s} \mathbf{W}^{T} \mathbf{H}^{T}+\mathbf{R}_{n}\right)-2 \mathbf{R}_{s}^{T} \mathbf{W}^{T} \mathbf{H}^{T}=0 .
$$

Thus, the optimal equalizer, denoted by $\mathbf{G}^{*}$, is given by

$$
\mathbf{G}^{*}=\mathbf{R}_{s}^{T} \mathbf{W}^{T} \mathbf{H}^{T}\left(\mathbf{H} \mathbf{W} \mathbf{R}_{s} \mathbf{W}^{T} \mathbf{H}^{T}+\mathbf{R}_{n}\right)^{-1} .
$$

Substituting (9) into (4), we can obtain the new MSE expression, given by $\mathrm{MSE}^{\prime}=\operatorname{tr}\left\{\left(\mathbf{W}^{T} \mathbf{H}^{T} \mathbf{R}_{n}^{-1} \mathbf{H W}+\mathbf{R}_{s}^{-1}\right)^{-1}\right\}$. Thus, the optimization problem of (6) can be reformulated as

$$
\min _{\mathbf{W}} f(\mathbf{W})=\mathrm{MSE}^{\prime} \text {, s.t. (7). }
$$

Next, we will invoke the PG approach of [8] to find an efficient solution of the problem (10). Note that the PG iteratively solves a constrained optimization. In each iteration, we update $\widehat{\mathbf{W}}^{n}$ by moving $\mathbf{W}^{n-1}$ along the negative gradient direction using an appropriate step size:

$$
\widehat{\mathbf{W}}^{n}=\mathbf{W}^{n-1}-\alpha_{n} \nabla f\left(\mathbf{W}^{n-1}\right), n=1,2, \cdots .
$$

Here, $\mathbf{W}^{n-1}$ represents the TPC matrix at the $n$th iteration, $\alpha_{n}$ is the step size, and $\nabla f(\mathbf{W})$ denotes the gradient of $f(\mathbf{W})$ w.r.t. $\mathbf{W}$, given as $\nabla f(\mathbf{W})=-2 \mathbf{H}^{T} \mathbf{R}_{n}^{-1} \mathbf{H W}\left(\mathbf{U}^{2}\right)^{-1}$, where $\mathbf{U}=\mathbf{W}^{T} \mathbf{H}^{T} \mathbf{R}_{n}^{-1} \mathbf{H W}+\mathbf{R}_{s}^{-1}$. The detailed derivations are given in Appendix A.

Based on the philosophy of PG, we then find the feasible $\mathbf{W}^{n}$ via projecting $\widehat{\mathbf{W}}^{n}$ onto the feasible region. We solve

$$
\min _{\mathbf{W}^{n}}\left\|\mathbf{W}^{n}-\widehat{\mathbf{W}}^{n}\right\|_{\mathrm{F}}^{2}
$$

s.t. $\operatorname{abs}\left\{\mathbf{W}^{n}\right\} \boldsymbol{\Delta}+\mathbf{W}^{n} \widehat{\mathbf{b}} \leq \widehat{\mathbf{p}}_{L}, \operatorname{abs}\left\{\mathbf{W}^{n}\right\} \boldsymbol{\Delta}-\mathbf{W}^{n} \widehat{\mathbf{b}} \leq \widehat{\mathbf{p}}_{H}$,

where $\|\cdot\|_{F}$ is the Frobenius norm of a matrix. Observing (12), the problem can be equivalently divided into the following $N_{t}$ subproblems:

$$
\begin{aligned}
& \min _{\mathbf{w}_{i}^{n}}\left\|\mathbf{w}_{i}^{n}-\widehat{\mathbf{w}}_{i}^{n}\right\|_{2}^{2}, \\
& \text { s.t. } \operatorname{abs}\left\{\mathbf{w}_{i}^{n}\right\} \boldsymbol{\Delta}+\mathbf{w}_{i}^{n} \widehat{\mathbf{b}} \leq \widehat{p}_{L, i}, \text { abs }\left\{\mathbf{w}_{i}^{n}\right\} \boldsymbol{\Delta}-\mathbf{w}_{i}^{n} \widehat{\mathbf{b}} \leq \widehat{p}_{H, i},
\end{aligned}
$$

where $\|\cdot\|_{2}$ denotes the $l_{2}$ norm of a vector, $\mathbf{w}_{i}^{n}$ and $\widehat{\mathbf{w}}_{i}^{n}$ are the $i$ th row of $\mathbf{W}^{n}$ and $\widehat{\mathbf{W}}^{n}, \widehat{p}_{L, i}$ and $\widehat{p}_{H, i}$ are the $i$ th element of $\widehat{\mathbf{p}}_{L}$ and $\widehat{\mathbf{p}}_{H}$. The above problem fortunately does have a closed-form solution, as formulated in Theorem 1 .

Theorem 1: When $\widehat{\mathrm{w}}_{i}^{n}$ satisfies the constraint in (13), the solution of (13) is $\mathbf{w}_{i}^{n}=\widehat{\mathbf{w}}_{i}^{n}$. Otherwise, the solution of (13) is given by

$$
w_{i j}^{n}=\operatorname{sign}\left\{\widehat{w}_{i j}^{n}\right\}\left(\left|\widehat{w}_{i j}^{n}\right|-\lambda_{1} r_{i j}-\lambda_{2} u_{i j}\right)^{+} .
$$

Here, $\operatorname{sign}\{x\}$ denotes the sign of $x,(x)^{+}=\max \{x, 0\}, w_{i j}^{n}$ and $\widehat{w}_{i j}^{n}$ are the jth elements of $\mathbf{w}_{i}^{n}$ and $\widehat{\mathbf{w}}_{i}^{n}$, respectively, while $r_{i j}=\frac{1}{2} \Delta_{j}+\frac{1}{2} \operatorname{sign}\left\{\widehat{w}_{i j}^{n}\right\} \widehat{b}_{j}$ and $u_{i j}=\frac{1}{2} \Delta_{j}-\frac{1}{2} \operatorname{sign}\left\{\widehat{w}_{i j}^{n}\right\} \widehat{b}_{j}$, and $\lambda_{1}$ and $\lambda_{2}$ are the Lagrange multipliers, which can be obtained through the following steps:

1. Solve $g_{\lambda_{1}}^{\prime}\left(\lambda_{1}, 0\right)=0$ to obtain the solution, denoted by $\lambda_{1}=\alpha_{1}$. If $g_{\lambda_{2}}^{\prime}\left(\alpha_{1}, 0\right) \leq 0$, then set $\lambda_{1}=\alpha_{1}$ and $\lambda_{2}=0$, and stop. Otherwise, go to next step.

2. Solve $g_{\lambda_{2}}^{\prime}\left(0, \lambda_{2}\right)=0$ to obtain the solution, denoted by $\lambda_{2}=\alpha_{2}$. If $g_{\lambda_{1}}^{\prime}\left(0, \alpha_{2}\right) \leq 0$, then set $\lambda_{1}=0$ and $\lambda_{2}=\alpha_{2}$, and stop. Otherwise, go to next step.

3. For $\lambda_{2} \in\left[0, \alpha_{2}\right]$, solve $h_{1}\left(\lambda_{2}\right)-h_{2}\left(\lambda_{2}\right)=0$ through the bisection method to obtain the solution, denoted by $\lambda_{2}=$ $\alpha_{3}$. Then, $\lambda_{1}=h_{1}\left(\alpha_{3}\right)$ and $\lambda_{2}=\alpha_{3}$. 
The functions $g_{\lambda_{1}}^{\prime}\left(\lambda_{1}, \lambda_{2}\right)$ and $g_{\lambda_{2}}^{\prime}\left(\lambda_{1}, \lambda_{2}\right)$ are defined as

$$
\left\{\begin{aligned}
g_{\lambda_{1}}^{\prime}\left(\lambda_{1}, \lambda_{2}\right) & =\sum_{j=1}^{K} 2 r_{i j}\left(\left|\widehat{w}_{i j}^{n}\right|-\lambda_{1} r_{i j}-\lambda_{2} u_{i j}\right)^{+}-\widehat{p}_{L, i}, \\
g_{\lambda_{2}}^{\prime}\left(\lambda_{1}, \lambda_{2}\right) & =\sum_{j=1}^{K} 2 u_{i j}\left(\left|\widehat{w}_{i j}^{n}\right|-\lambda_{1} r_{i j}-\lambda_{2} u_{i j}\right)^{+}-\widehat{p}_{H, i} .
\end{aligned}\right.
$$

The functions $\lambda_{1}=h_{1}\left(\lambda_{2}\right)$ and $\lambda_{1}=h_{2}\left(\lambda_{2}\right)$ are defined based on $g_{\lambda_{1}}^{\prime}\left(\lambda_{1}, \lambda_{2}\right)=0$ and $g_{\lambda_{2}}^{\prime}\left(\lambda_{1}, \lambda_{2}\right)=0$, respectively.

Proof: See Appendix B.

Now we are ready to summarize the procedure of the proposed joint dimming control and transceiver design method in Algorithm 1. The corresponding convergence analysis can be found in [8].

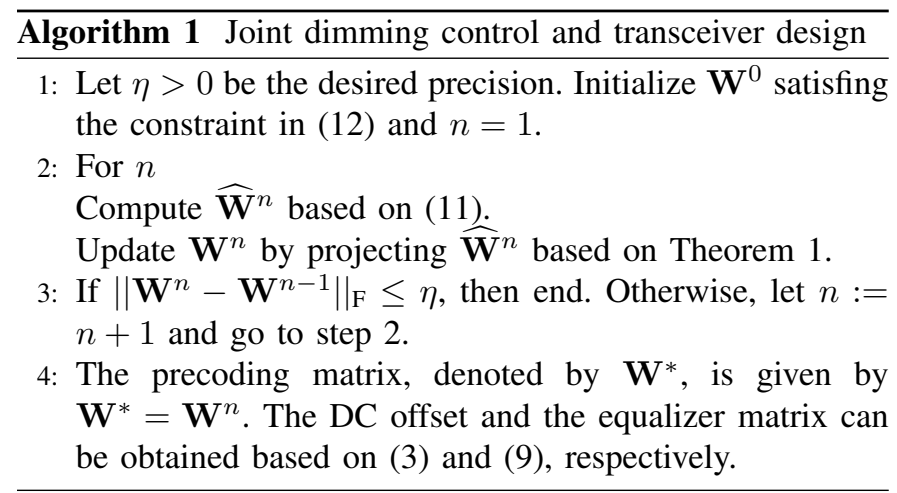

\section{NuMERICAL RESUlts AND Discussions}

In this section, our numerical results are presented for evaluating the performance of the proposed transceiver. As shown in Fig. 1, we consider a $(4 \times 4)$ MIMO VLC system, which is comprised of four LED arrays distributed uniformly, and a single receiver having four PDs arranged in a $2 \times 2$ array on a $0.1 \mathrm{~m}$ pitch [1], [3]. Each LED array has $3600(60 \times 60)$ LEDs, and the linear dynamic range of a single LED is limited to $1 \sim 10 \mathrm{~mW}$. The other relevant parameters of the VLC system are set to the same values as in [1], hence they are not explicitly listed here.

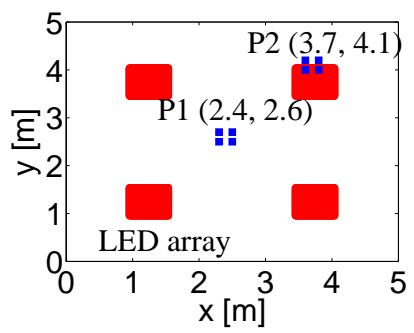

Fig. 1. The room model. The room size is $5 \mathrm{~m} \times 5 \mathrm{~m} \times 3 \mathrm{~m}$. The height of the receiver is $0.85 \mathrm{~m}$ from the floor. The height of LED arrays are $2.5 \mathrm{~m}$ from the floor.

In Fig. 2, we present the BER of the MIMO VLC system, when the receiver is located at positions $\mathrm{P} 1$ and $\mathrm{P} 2$, as depicted in Fig. 1. In the simulations, both 8-PPM and 8-PAM are used for position P1, while 4-PPM and 4-PAM are used for position $\mathrm{P} 2$. For PPM, the precoder matrix is applied to each time
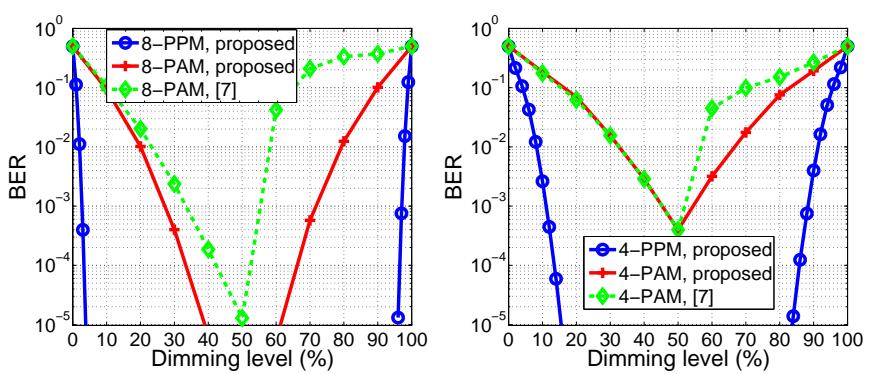

Fig. 2. BER of the MIMO VLC system with $K=3$ and $\eta=10^{-2}$ (left: $\mathrm{P} 1$, right: $\mathrm{P} 2$ ).

slot, and $\mathbf{R}_{s}$ is generated slot by slot. The performance of the transceiver of [7] using bipolar PAM which has zero mean is also provided for comparison. Since [7] considered only the non-negative nature of the signals in its design, the LED nonlinearity may lead to the non-linear clipping of the signals at relatively high dimming level. It can be observed that the proposed transceiver outperforms that of [7] in terms of its BER, especially at high dimming level. Additionally, the BER performance becomes better when the dimming level tends towards $50 \%$. Under relatively low or high dimming levels, the transceiver employing PAM yields a poor performance, while the transceiver employing PPM still performs well. Therefore, despite its low spectrum efficiency compared to PAM, PPM is a beneficial design option providing a stable communication link at relatively low or high dimming levels.

Fig. 3 illustrates the convergence behavior of our algorithms. It can be clearly observed that the proposed design achieves a much higher convergence speed than the algorithm of [7]. Moreover, each iteration of the proposed method relies on closed-form expressions, which results in a simple solution without the need for any of the special optimization software packages used in [7].

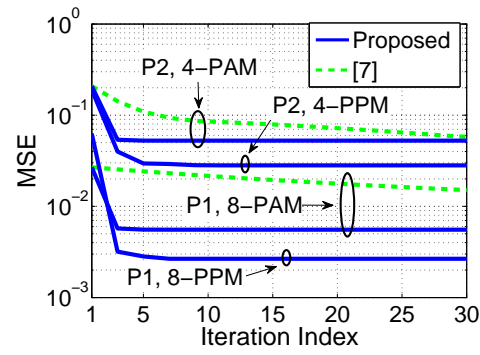

Fig. 3. Convergence of the proposed algorithms with dimming level $\rho=0.4$.

\section{CONCLUSIONS}

A novel transceiver combined with dimming control was developed for MIMO VLC, which is capable of minimizing the MSE between the transmitted and received signals, while maintaining the target indoor illumination level. The simulation results demonstrate that the BER performance of the proposed transceiver is improved as the dimming level tends to $50 \%$. Additionally, despite its low spectrum efficiency compared to PAM, PPM is a beneficial design option in VLC at relatively low or high dimming levels. 


\section{APPENDIX A \\ DERIVATIVE OF $\nabla f(\mathbf{W})$}

Based on the definition of $\mathbf{U}$, we have $h(\mathbf{U})=$ $f(\mathbf{W})=\operatorname{tr}\left\{\mathbf{U}^{-1}\right\}$. According to the Chain rule of [10], the derivative of $f(\mathbf{W})$ w.r.t. $w_{i j}$ can be calculated as $\partial f(\mathbf{W}) / \partial w_{i j}=\operatorname{tr}\left\{[\partial h(\mathbf{U}) / \partial \mathbf{U}]^{T} \partial \mathbf{U} / \partial w_{i j}\right\}$. From [10, Chap. 2], we have $\partial h(\mathbf{U}) / \partial \mathbf{U}=-\left(\mathbf{U}^{2}\right)^{-1}$ and $\partial \mathbf{U} / \partial w_{i j}=\mathbf{W}^{T} \mathbf{H}^{T} \mathbf{R}_{n}^{-1} \mathbf{H} \mathbf{J}^{i j}+\left(\mathbf{W}^{T} \mathbf{H}^{T} \mathbf{R}_{n}^{-1} \mathbf{H} \mathbf{J}^{i j}\right)^{T}$, where $\mathbf{J}^{i j}$ denotes the matrix having a single " 1 " in the $i$ th row and $j$ th column, whilst zeros elsewhere. Therefore, $\partial f(\mathbf{W}) / \partial w_{i j}=-\left[2\left(\mathbf{U}^{2}\right)^{-1} \mathbf{W}^{T} \mathbf{H}^{T} \mathbf{R}_{n}^{-1} \mathbf{H}\right]_{j i}$, where $[\mathbf{X}]_{j i}$ denotes the $(j, i)$-th element of $\mathbf{X}$. Accordingly, the gradient of $f(\mathbf{W})$ is given by $\nabla f(\mathbf{W})=$ $-2 \mathbf{H}^{T} \mathbf{R}_{n}^{-1} \mathbf{H W}\left(\mathbf{U}^{2}\right)^{-1}$.

\section{APPENDIX B \\ PROOF OF THEOREM 1}

When $\widehat{\mathbf{w}}_{i}^{n}$ satisfies the constraint, the solution of (13) is directly obtained. Thus, we focus our attention on the scenario when $\widehat{\mathbf{w}}_{i}^{n}$ does not satisfy the constraint of (13). We can equivalently solve the problem of (13) via its dual problem, given by

$$
\begin{aligned}
g\left(\lambda_{1}, \lambda_{2}\right)= & \inf _{\mathbf{w}_{i}^{n}}\left\|\mathbf{w}_{i}^{n}-\widehat{\mathbf{w}}_{i}^{n}\right\|_{2}^{2}+\lambda_{1}\left[\operatorname{abs}\left\{\mathbf{w}_{i}^{n}\right\} \boldsymbol{\Delta}+\mathbf{w}_{i}^{n} \mathbf{b}\right. \\
& \left.-\widehat{p}_{L, i}\right]+\lambda_{2}\left[\operatorname{abs}\left\{\mathbf{w}_{i}^{n}\right\} \boldsymbol{\Delta}-\mathbf{w}_{i}^{n} \mathbf{b}-\widehat{p}_{H, i}\right] \\
\triangleq & \sum_{j=1}^{K} g_{j}\left(\lambda_{1}, \lambda_{2}\right)-\lambda_{1} \widehat{p}_{L, i}-\lambda_{2} \widehat{p}_{H, i}
\end{aligned}
$$

where $\lambda_{1}$ and $\lambda_{2}$ are the Lagrange multipliers, and $g_{j}\left(\lambda_{1}, \lambda_{2}\right)$ is defined as

$$
\begin{aligned}
g_{j}\left(\lambda_{1}, \lambda_{2}\right)= & \inf _{w_{i j}^{n}}\left(w_{i j}^{n}-\widehat{w}_{i j}^{n}\right)^{2}+\left(\lambda_{1}+\lambda_{2}\right)\left|w_{i j}^{n}\right| \Delta_{j} \\
& +\left(\lambda_{1}-\lambda_{2}\right) w_{i j}^{n} b_{j} .
\end{aligned}
$$

We analyze (17) for two different cases, i.e. for $w_{i j}^{n}>0$ and $w_{i j}^{n} \leq 0$, in order to find the minimum of the problem. It may be readily shown that $g_{j}\left(\lambda_{1}, \lambda_{2}\right)$ can be expressed as

$$
g_{j}\left(\lambda_{1}, \lambda_{2}\right)=\left(\widehat{w}_{i j}^{n}\right)^{2}-\left[\left(\left|\widehat{w}_{i j}^{n}\right|-\lambda_{1} r_{i j}-\lambda_{2} u_{i j}\right)^{+}\right]^{2},
$$

which corresponds to the optimal $w_{i j}^{n}$ given by (14). Now the dual problem of (13) can be formulated as

$$
\max _{\lambda_{1}, \lambda_{2}} g\left(\lambda_{1}, \lambda_{2}\right), \text { s.t. } \lambda_{1} \geq 0, \lambda_{2} \geq 0 .
$$

The Karush-Kuhn-Tucker (KKT) conditions of the above dual problem are given by

$$
\begin{aligned}
& g_{\lambda_{1}}^{\prime}\left(\lambda_{1}, \lambda_{2}\right)+\nu_{1}=0, \nu_{1} \geq 0, \quad \nu_{1} \lambda_{1}=0, \quad \lambda_{1} \geq 0, \\
& g_{\lambda_{2}}^{\prime}\left(\lambda_{1}, \lambda_{2}\right)+\nu_{2}=0, \quad \nu_{2} \geq 0, \quad \nu_{2} \lambda_{2}=0, \quad \lambda_{2} \geq 0 .
\end{aligned}
$$

where $\nu_{1}$ and $\nu_{2}$ are the Lagrange multipliers of the problem in (19), $g_{\lambda_{1}}^{\prime}\left(\lambda_{1}, \lambda_{2}\right)$ and $g_{\lambda_{2}}^{\prime}\left(\lambda_{1}, \lambda_{2}\right)$ are the partial derivatives of $g\left(\lambda_{1}, \lambda_{2}\right)$ w.r.t. $\lambda_{1}$ and $\lambda_{2}$, respectively, given as (15).

It is observed that $\lambda_{1}=0$ and $\lambda_{2}=0$ cannot satisfy the conditions in (20). Therefore, we discuss the above KKT conditions in the following three cases:
Case 1: when $\lambda_{1}>0$ and $\lambda_{2}=0$, the conditions are simplified to $g_{\lambda_{1}}^{\prime}\left(\lambda_{1}, 0\right)=0, g_{\lambda_{2}}^{\prime}\left(\lambda_{1}, 0\right) \leq 0$.

Case 2: when $\lambda_{1}=0$ and $\lambda_{2}>0$, the conditions are rewritten as $g_{\lambda_{1}}^{\prime}\left(0, \lambda_{2}\right) \leq 0, g_{\lambda_{2}}^{\prime}\left(0, \lambda_{2}\right)=0$.

Case 3: when $\lambda_{1}>0$ and $\lambda_{2}>0, \lambda_{1}$ and $\lambda_{2}$ satisfy $g_{\lambda_{1}}^{\prime}\left(\lambda_{1}, \lambda_{2}\right)=0, g_{\lambda_{2}}^{\prime}\left(\lambda_{1}, \lambda_{2}\right)=0$.

For Case 1, we can first solve $g_{\lambda_{1}}^{\prime}\left(\lambda_{1}, 0\right)=0$ to obtain the solution, denoted by $\lambda_{1}=\alpha_{1}$. Note that we can express $g_{\lambda_{1}}^{\prime}\left(\lambda_{1}, 0\right)$ as a piecewise function of $\lambda_{1}$, and find it is a continuous decreasing function w.r.t. $\lambda_{1}$. Since $g_{\lambda_{1}}^{\prime}(0,0)>0$ and $g_{\lambda_{1}}^{\prime}(+\infty, 0)<0, g_{\lambda_{1}}^{\prime}\left(\lambda_{1}, 0\right)=0$ always has a positive solution, i.e., $\alpha_{1}>0$. Then if $g_{\lambda_{2}}^{\prime}\left(\alpha_{1}, 0\right) \leq 0, \lambda_{1}=\alpha_{1}$ and $\lambda_{2}=0$ can satisfy the KKT conditions. Otherwise, we continue to use the same way to analyze Case 2.

If the conditions of the first two cases do not hold, then we need to solve the equations in Case 3. We first define two functions based on $g_{\lambda_{1}}^{\prime}\left(\lambda_{1}, \lambda_{2}\right)=0$ and $g_{\lambda_{2}}^{\prime}\left(\lambda_{1}, \lambda_{2}\right)=$ 0 , denoted by $\lambda_{1}=h_{1}\left(\lambda_{2}\right)$ and $\lambda_{1}=h_{2}\left(\lambda_{2}\right)$, respectively. Then, we can obtain the solution of the equations in Case 3 by solving $h_{1}\left(\lambda_{2}\right)-h_{2}\left(\lambda_{2}\right)=0$. For any number $\varepsilon>0$, there always exists $\delta=\min \left\{r_{i j} / u_{i j}\right\} \varepsilon$ such that for $\lambda_{2}$ satisfying $\left|\lambda_{2}-c\right|<\delta, h_{1}\left(\lambda_{2}\right)^{j}$ satisfies $\left|h_{1}\left(\lambda_{2}\right)-h_{1}(c)\right|<\varepsilon$, which can be proved through proof by contradiction. Therefore, $h_{1}\left(\lambda_{2}\right)$ is a continuous function. Similarly, we can also prove that $h_{2}\left(\lambda_{2}\right)$ is a continuous function. Since the conditions in Case 1 and Case 2 do not hold, we have $g_{\lambda_{2}}^{\prime}\left(\alpha_{1}, 0\right)>0$ and $g_{\lambda_{1}}^{\prime}\left(0, \alpha_{2}\right)>$ 0 , implying $h_{2}(0)>\alpha_{1}$ and $h_{1}\left(\alpha_{2}\right)>0$. Therefore, $h_{1}(0)-$ $h_{2}(0)=\alpha_{1}-h_{2}(0)<0$ and $h_{1}\left(\alpha_{2}\right)-h_{2}\left(\alpha_{2}\right)=h_{1}\left(\alpha_{2}\right)>0$. Consequently, $h_{1}\left(\lambda_{2}\right)-h_{2}\left(\lambda_{2}\right)=0$ always has a solution for $\lambda_{2} \in\left[0, \alpha_{2}\right]$, which can be obtained by the bisection method.

\section{REFERENCES}

[1] T. Komine and M. Nakagawa, "Fundamental analysis for visible-light communication system using LED lights," IEEE Trans. Consum. Electron., vol. 50, no. 1, pp. 100-107, Feb. 2004.

[2] F. Zafar, D. Karunatilaka, and R. Parthiban, "Dimming schemes for visible light communication: The state of research," IEEE Wireless Commun., vol. 22, no. 2, pp. 29-35, Apr. 2015.

[3] L. Zeng, D. O'Brien, H. Minh, G. Faulkner, K. Lee, D. Jung, Y. Oh, and E. T. Won, "High data rate multiple input multiple output (MIMO) optical wireless communications using white LED lighting," IEEE $J$. Sel. Areas Commun., vol. 27, no. 9, pp. 1654-1662, Dec. 2009.

[4] A. Burton, H. L. Minh, Z. Ghassemlooy, E. Bentley, and C. Botella, "Experimental demonstration of $50-\mathrm{Mb} / \mathrm{s}$ visible light communications using $4 \times 4$ MIMO," IEEE Photon. Technol. Lett., vol. 26, no. 9, pp. 945-948, May 2014.

[5] A. Nuwanpriya, S.-W. Ho, and C. S. Chen, "Indoor MIMO visible light communications: Novel angle diversity receivers for mobile users," IEEE J. Sel. Areas Commun., vol. 33, no. 9, pp. 1780-1792, Sept. 2015.

[6] K.-H. Park, Y.-C. Ko, and M. Alouini, "On the power and offset allocation for rate adaptation of spatial multiplexing in optical wireless MIMO channels," IEEE Trans. Commun., vol. 61, no. 4, pp. 1535-1543, Apr. 2013.

[7] K. Ying, H. Qian, R. Baxley, and S. Yao, "Joint optimization of precoder and equalizer in MIMO VLC systems," IEEE J. Sel. Areas Commun., vol. 33, no. 9, pp. 1949-1958, Sept. 2015.

[8] P. H. Calamai and J. J. Moré, "Projected gradient methods for linearly constrained problems," Mathematical programming, vol. 39, no. 1, pp. 93-116, Mar. 1987.

[9] Q. Wang, Z. Wang, and L. Dai, "Asymmetrical hybrid optical OFDM for visible light communications with dimming control," IEEE Photon. Technol. Lett., vol. 27, no. 9, pp. 974-977, May 2015.

[10] K. B. Petersen and M. S. Pedersen, The Matrix Cookbook. Technical University of Denmark, 2012. 